\title{
The effect of polymeric additives on the structure and permeability of poly(vinyl alcohol) asymmetric membranes
}

\author{
W.-Y. Chuang ${ }^{\mathrm{a}}$, T.-H. Young ${ }^{\mathrm{b}} *$, W.-Y. Chiu ${ }^{\mathrm{a}}$, C.-Y. Lin ${ }^{\mathrm{c}}$ \\ ${ }^{\mathrm{a}}$ Institute of Materials Science and Engineering, College of Engineering, National Taiwan University, Taipei 10016, Taiwan, ROC \\ ${ }^{\mathrm{b}}$ Institute of Biomedical Engineering, College of Medicine and College of Engineering, National Taiwan University, Taipei 10016, Taiwan, ROC \\ ${ }^{\mathrm{c}}$ Advanced Instrumentation Center, National Taiwan University, Taipei, Taiwan, ROC
}

Received 4 June 1999; received in revised form 30 September 1999; accepted 26 October 1999

\begin{abstract}
The manufacture and dextran-rejection properties of asymmetric poly(vinyl alcohol) (PVA) membranes have been described in this study. Membranes were prepared from a casting solution of PVA, water as solvent, and water-soluble polymeric additive by immersing them in $\mathrm{Na}_{2} \mathrm{SO}_{4} / \mathrm{KOH} / \mathrm{H}_{2} \mathrm{O}$ as coagulant medium. Experiments showed that the dextran and poly(vinyl pyrrolidone) (PVP) additives exerted a different influence on the structure and permeability of membranes. Especially, the structure of skin layer strongly depended on the polymeric additives in the casting solution. The addition of dextran additives in the system could induce pores in the top layer. Conversely, the PVP additives effectively blocked the interstitial cavities within the top layer to generate a more compact structure. A mechanism describing that the affinity between additive and casting solution as well as between additive and coagulant medium was proposed to investigate the effect of dextran and PVP additives in the formation of PVA membranes. The results presented here offer a better understanding of relationships between the membrane formation mechanism and the skin structure when designing an asymmetric membrane by the addition of polymeric additives in the casting solution. (C) 2000 Elsevier Science Ltd. All rights reserved.
\end{abstract}

Keywords: Poly(vinyl alcohol); Polymeric additive; Dextran

\section{Introduction}

Since the development of asymmetric type membrane by Loeb and Sourirajan [1], considerable progress has been made in various aspects of membrane-related fields [2]. The interest in studies of membranes is due to the necessity of membranes with different separation properties. An asymmetrical membrane is characterized by a thin and dense top layer, commonly recognized as the skin layer, and underneath which is a porous solid matrix. It is well known that the skin layer provides major resistance to the permeation of solute through the membrane, whereas the porous region functions exclusively as a mechanical support. The capability of an asymmetric membrane to reject or to admit a certain solute species is, therefore, determined by the morphology, the pore size and the dense degree of the skin layer. Accordingly, it is necessary to prepare asymmetric membranes with controllable

\footnotetext{
* Corresponding author. Tel.: +886-2-2397-0800, ext. 1455; fax: +8862-2394-0049.

E-mail address: thyoung @ha.mc.ntu.edu.tw (T.-H. Young).
}

structure of skin layer to achieve the required performance.

Being a material with good chemical stability, thermal stability and high water permeability, poly(vinyl alcohol) (PVA) seems an attractive material for producing membranes [3]. In order to control the membrane structure, low molecular weight component or the secondary polymer is frequently used as the additive in the membrane forming system [4] because it offers a convenient and effective way to develop membranes with high performances. We have reported that the applicability of PVA membranes for the separation of proteins by the addition of poly(ethylene glycol) in the casting solution to control the structure of skin layer [5,6]. This article is a continuation of publications to obtain more information about the effect of polymer additives on the structure and the permeability of PVA membranes. Instead of poly(ethylene glycol), a series of asymmetric membranes were prepared by adding dextran and poly(vinyl pyrrolidone) (PVP) to the casting solution. Experiments showed that the dextran and PVP additives exerted a different influence on the structure and permeability of membranes. Thus, a mechanism describing the 
Table 1

Composition of casting solution and permeation flux of membranes

\begin{tabular}{llllll}
\hline Membrane & $\begin{array}{l}\text { PVA } \\
(\mathrm{g})\end{array}$ & $\begin{array}{l}\mathrm{H}_{2} \mathrm{O} \\
(\mathrm{g})\end{array}$ & $\begin{array}{l}\text { Dextran } \\
(\mathrm{g})\end{array}$ & $\begin{array}{l}\text { PVP } \\
(\mathrm{g})\end{array}$ & $\begin{array}{l}\text { Permeation flux } \\
\left(\times 10^{7}\right)(\mathrm{m} / \mathrm{s})\end{array}$ \\
\hline M-1 & 1.4 & 8.6 & 0 & 0 & 0.56 \\
D-1 & 1.4 & 8.6 & 0.1 & 0 & 0.66 \\
D-2 & 1.4 & 8.6 & 0.2 & 0 & 0.77 \\
D-3 & 1.4 & 8.6 & 0.3 & 0 & 1.07 \\
D-4 & 1.4 & 8.6 & 0.4 & 0 & 2.28 \\
P-1 & 1.4 & 8.6 & 0 & 0.1 & 0.54 \\
P-2 & 1.4 & 8.6 & 0 & 0.2 & 0.52 \\
P-3 & 1.4 & 8.6 & 0 & 0.3 & 0.50 \\
P-4 & 1.4 & 8.6 & 0 & 0.4 & 0.48 \\
\hline
\end{tabular}

affinity between additive and casting solution as well as between additive and coagulant medium in the membrane formation was proposed for a better understanding of the role played by the polymeric additives.

\section{Experimental}

\subsection{Materials}

All reagents used in the experiment were of reagent grade, unless otherwise described. The membrane material, PVA having an average molecular weight of 74,800 was purchased from Chang Chun (Taiwan). Dextran with an average molecular weight of 12,000 (Sigma) and PVP with an average molecular weight of 10,000 (Fluka) were employed as additives. Water was double distilled and de-ionized before use.

\subsection{Membrane preparation}

Membranes were prepared by the immersion-precipitation technique. The polymer solution was prepared by dissolving $1.4 \mathrm{~g}$ of dried powder samples of PVA in $8.6 \mathrm{~g}$ of water at $90^{\circ} \mathrm{C}$. Subsequently, different amounts of additives were added to the PVA solution, as shown in Table 1. All membranes were cast from PVA solution at $25^{\circ} \mathrm{C}$ on a glass plate with an uniform thickness of about $100 \mu \mathrm{m}$ followed by precipitating the casting solution in a coagulation bath with $\mathrm{Na}_{2} \mathrm{SO}_{4} / \mathrm{KOH} / \mathrm{H}_{2} \mathrm{O}=$ saturate $/ 75 \mathrm{~g} / 1000 \mathrm{ml}$ [3].

\subsection{Scanning electron microscopy}

The morphologies of different faces of the membranes were examined using scanning electron microscopy (SEM). The membranes were freeze dried, then frozen in liquid nitrogen and fractured to expose the cross-sectional areas. The dried sample were gold coated and viewed with SEM (S-800, Hitachi, Japan) at $10 \mathrm{kV}$.

\subsection{Dextran permeation experiment}

Solute rejection and permeation flux were determined
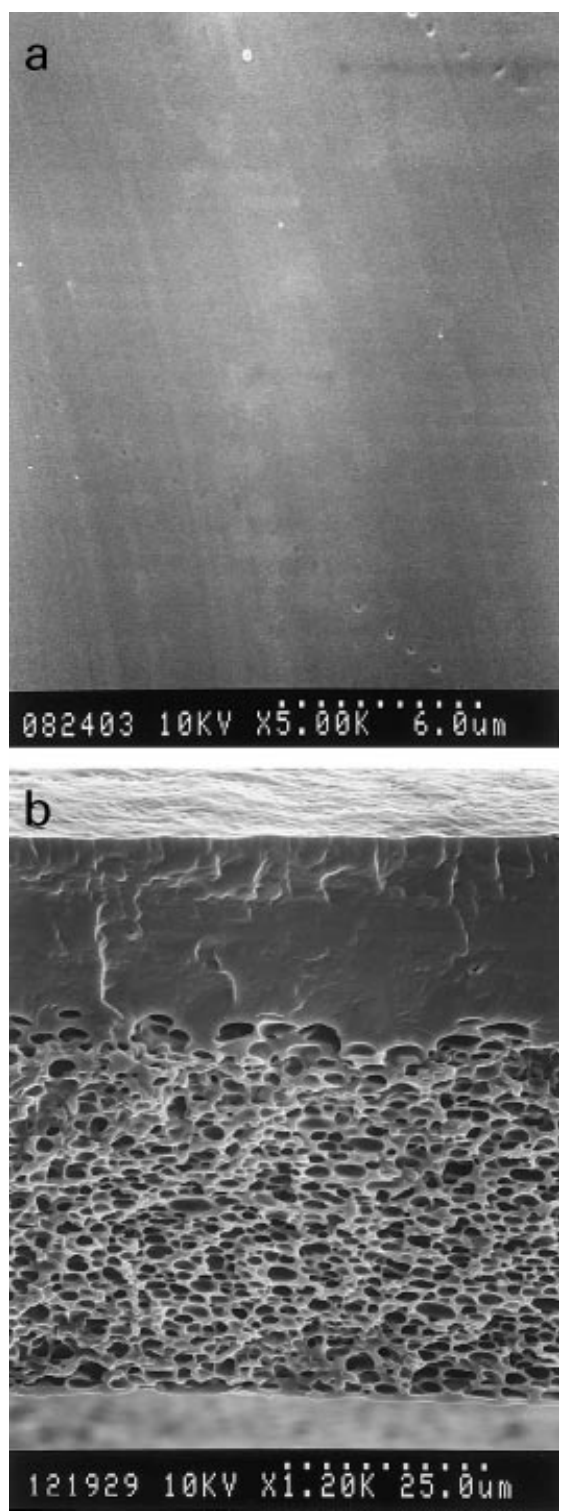

Fig. 1. SEM photographs of M-1 membrane: (a) top surface and (b) crosssection.

using a $43 \mathrm{~mm}$ dia. Amicon Stirred Ultrafiltration Cell (Model 8050). The solutes used in this work were dextrans with various molecular weights in the range of $6 \times 10^{3}-2 \times$ $10^{6}$ (Sigma). In the filtration process, low concentration feed dissolved in water $(1000 \mathrm{ppm})$ under a low pressure of $0.2 \mathrm{kgf} / \mathrm{cm}^{2}$ with vigorous agitation $(600 \mathrm{rpm})$ is to minimize the effect of concentration polarization [7,8]. After the permeating flux reaches a stable constant value (ca. $30 \mathrm{~min}$. after operation), samples of permeate were collected for subsequent chromatographic analysis by gel permeation chromatography (GPC) using a Waters 510 pump (USA) coupled with Ultrahydrogel SEC columns and a Waters 410 refractive index detector. The experimentally obtained rejection, $R$, was defined as $R=\left(C_{\mathrm{b}}-C_{\mathrm{p}}\right) / C_{\mathrm{b}}$, where $C_{\mathrm{b}}$ and $C_{\mathrm{p}}$ denote the concentration of bulk and permeated solutes, respectively. 

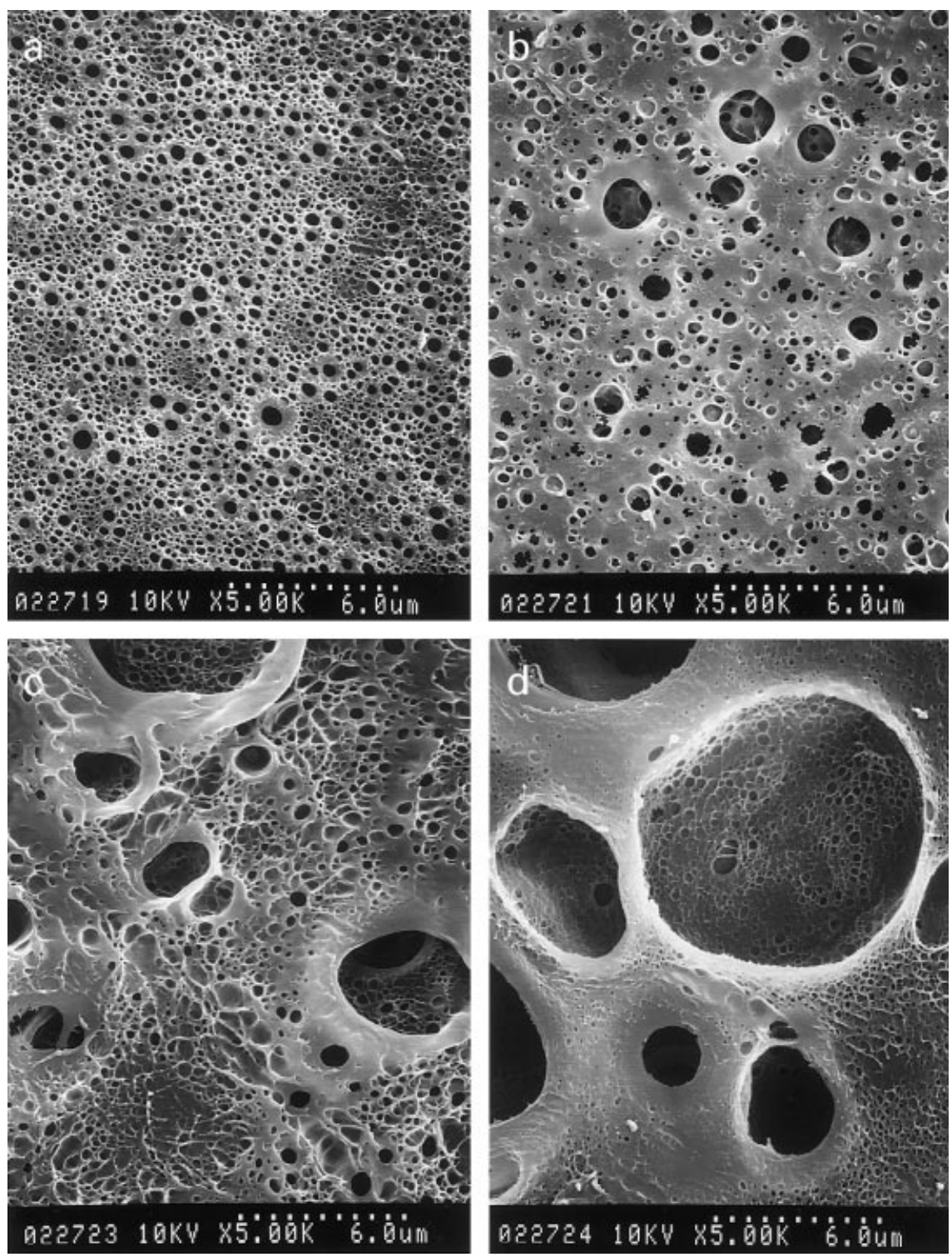

Fig. 2. SEM photographs of top surface of: (a) D-1; (b) D-2; (c) D-3; and (d) D-4 membranes.

\subsection{Light transmission experiment}

Light transmission experiments were performed to measure the precipitation rate of the immersion-precipitation process. The principle of light transmission experiments is that the light transmittance of the casting solution would decrease with the appearance of optical inhomogeneous. Therefore, the time that the light transmittance begins to drop can be used to represent the time of the onset of precipitation [9]. To carry out the light transmission experiment, an UV-lamp with green light was placed above the coagulation bath as light source and a light detector beneath the coagulation bath was used to measure the light transmittance. For detailed experimental set-up and procedures, one can refer to the work of Reuvers [10].

\section{Results and discussion}

\subsection{Membrane characterization}

Fig. 1 shows SEM photographs of M-1 membrane. The membrane was prepared from $14 \mathrm{wt} \%$ of PVA solution in the absence of polymeric additive. It is obvious that the membrane had an asymmetrical structure consisting of a dense skin layer and a porous sublayer that was occupied by cellular morphologies enclosed in polymer matrix. The skin layer is responsible for the permeation or rejection of solutes, whereas the porous bulk acts only as a mechanical support.

Membrane structure may be regulated by a variety of methods [2], one of which is the addition of the second polymer to the casting solution. As shown by Boom et al. 

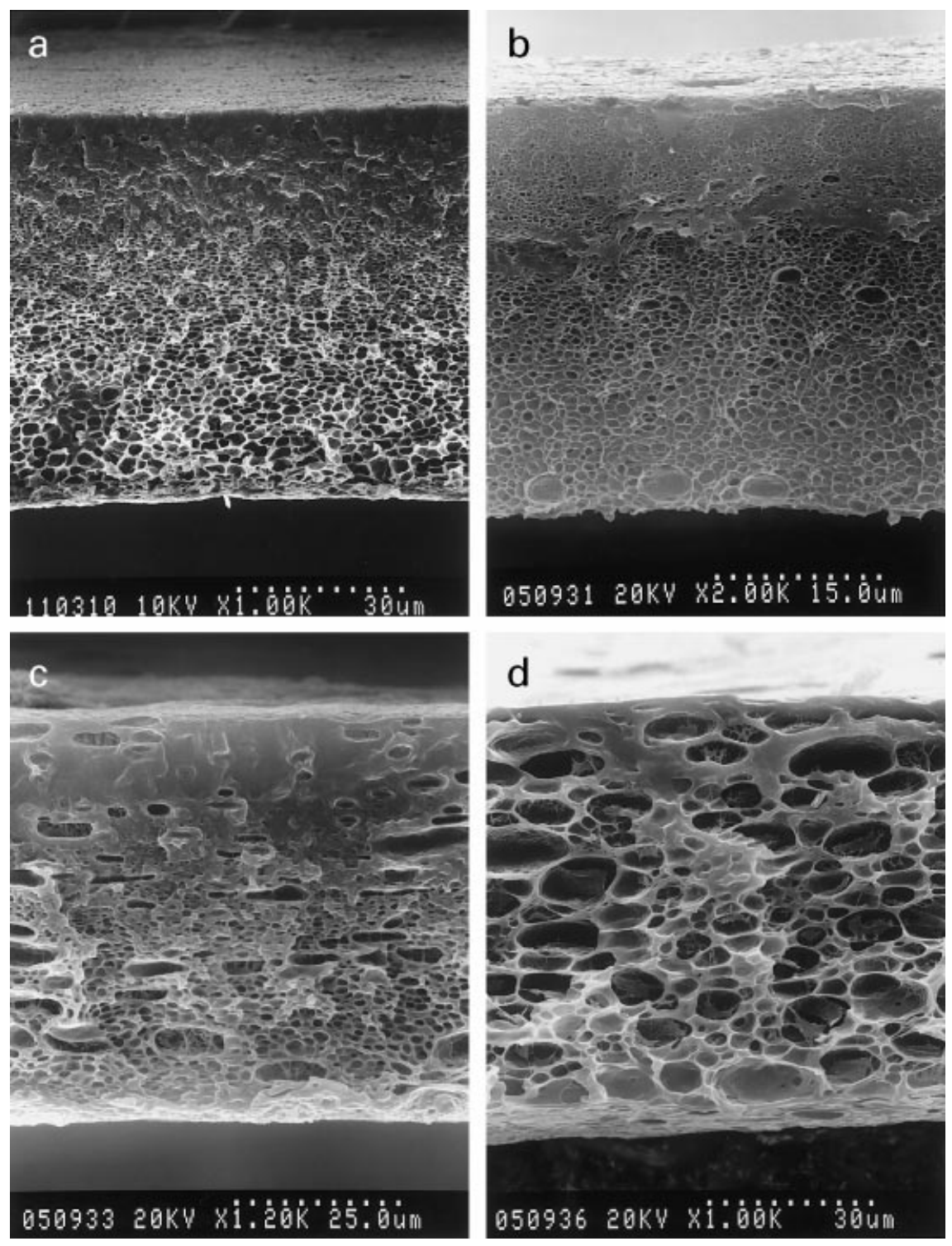

Fig. 3. SEM photographs of cross-section of: (a) D-1; (b) D-2; (c) D-3; and (d) D-4 membranes.

[11], the addition of PVP to the poly(ether sulfone) membrane system could suppress the formation of macrovoids in the sublayer, while the toplayer consisted of a closely packed layer of nodules. In this work, the effect of polymeric additive on the membrane morphology was investigated, especially on the membrane surface. After adding dextran to the casting solution, it could be observed that pores occurred in the skin layer (Figs. 2 and 3). The size of the pores increased with the increase in the amount of dextran in the casting solution. It should be noted that a small amount of dextran was enough to initiate the formation of pores in the skin layer. Since the skin layer dictates the permselectivity of a membrane, it is probable to have a strict control of the membrane performance by the addition of dextran in the casting solution, as will be described in the next paragraph. In addition, the change of the porous structure in support with the addition of dextran was observed as shown in Fig. 3. As compared with the cross section of membranes without the additive, the pore size appeared to somewhat swell in the support.

To estimate the performance of PVA membranes having a molecular sieve effect, the filtration experiment of a dextran solution through prepared membranes was carried out. Fig. 4 shows the rejection (or permeation) behaviour of PVA membranes with and without dextran additives in the casting solution. It could be observed that the rejection increased monotonically with the molecular weight of the solute, as in ordinary filtration processes. Furthermore, the rejection curves markedly shifted toward a high molecular weight side of dextran with the increase in the amount of dextran in the casting solution. In addition, the permeation flux of PVA membranes (Table 1) increased with increasing the amounts of the dextran additive in the casting solution. These results were consistent with qualitative observations 


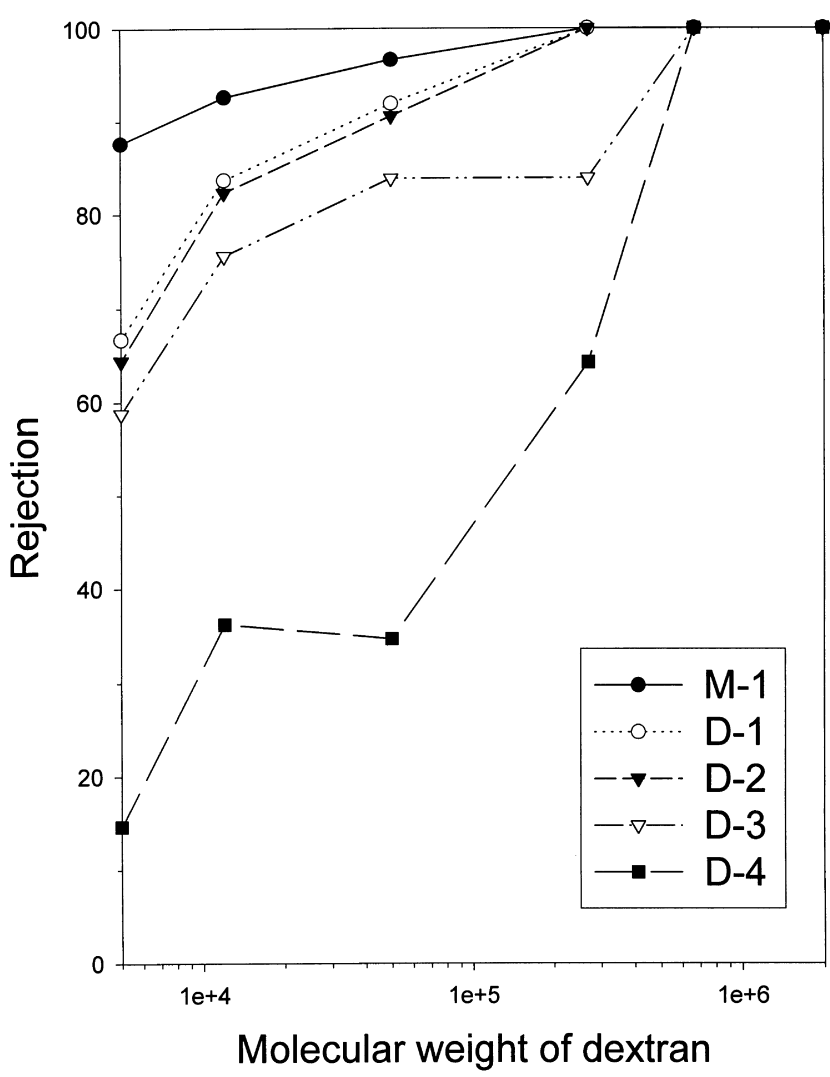

Fig. 4. Rejection curves of PVA membranes having various amounts of the dextran additive.

of SEM, suggesting that the dextran additives played a role of pore former in the skin layer to increase the permeability of the PVA membranes.

Young et al. [5] and Kobayashi et al. [12] proposed a formation mechanism of pores in the skin layer by watersoluble additives. After the membrane is formed, watersoluble additives are dissolved to create pores, which were originally occupied by additives in the incipient membrane. To examine if the pore-forming mechanism can be applied to other water-soluble additives, instead of dextran, PVP was added to the casting solution to prepare PVA membranes. Figs. 5 and 6 show the membrane structure made with varying concentrations of PVP additive. Since both dextran and PVP are water-soluble polymers, it is reasonable to expect that they have the same influence on the structure of PVA membranes. However, it appears that the addition of $1-4 \mathrm{wt} \%$ of PVP in the casting solution resulted in the absence of pores in the membrane surface, contradictory to our expectation. Similar to M-1 membrane, the obtained membranes with the PVP additive in the casting solution had a dense skin layer. Therefore, it seems that the formation of pores in the skin layer by the dextran additive cannot be explained by its water-soluble property.

Based on the above result, the rejection of dextran through membranes with PVP additives in the casting solution would be larger than that with dextran additives.
The effect of PVP additives on the filtration of a dextran solution is shown in Fig. 7. The rejection of dextran through membranes with PVP additive was not only larger than that with dextran additives but also larger than the M-1 membrane. This suggests that the membranes with PVP additives markedly restricted the permeation of dextran. In addition, with the increase in the PVP content in the casting solution, there was a tendency for the rejection of dextran through membranes to become large. Likewise, the permeation flux of PVA membranes (Table 1) decreased with increasing the amounts of the PVP additive in the casting solution. These indicate that the skin layer of the membranes became denser with the increase in the amount of PVP added. On basis of the above observation, it can be concluded that the dextran and PVP additives exerted a different influence on the structure and permeability of PVA membranes.

Another interesting characteristic of the effect of dextran and PVP additives is their precipitation rates during membrane formation were different as shown in Figs. 8 and 9. For each profile, the time at which light intensity began to rapidly decrease was identified as the onset point of precipitation. It appears that the so-called delayed precipitation took place in all the immersion cases. However, the role of PVP and dextran is contrary. The precipitation rate decreased with increasing the amount of PVP in the casting solution. Conversely, the addition of dextran in the casting solution caused a faster precipitating rate. In general, membranes with instantaneous precipitation rate often show macrovoids $[2,9]$. This implies that the PVA membrane with dextran additives in the casting solution had a tendency toward forming large pores by accelerating its precipitation rate. This can be related to the effect of the polymeric additives on the diffusivity of coagulant into the polymer solution $[13,14]$. Thus, the membrane formation mechanism may be changed and will be described in the next section.

\subsection{Membrane formation mechanism}

In order to understand the asymmetric membrane formation mechanism, it is convenient to analyze the membrane as a two-layer structure: the dense top layer and the porous sublayer [15]. Clearly, the top layer and the sublayer have different formation mechanism, leading therefore to different morphologies. With the casting solution immersed into the coagulation bath, the toplayer was formed first at the casting solution-coagulant interface. Due to the salt effect of sufficiently high concentration of ions in the coagulant bath, water in the casting solution rapidly desolvated into the coagulation bath when the casting solution and coagulant medium came to contact. Consequently, high polymer concentration resulting from water outflow was gradually set up. Such a high polymer concentration is expected to produce a dense skin layer on the M-1 membrane surface. Hence, the surface of the M-1 membrane was very hard and stiff so as to preclude the possibility of any porous morphology. 

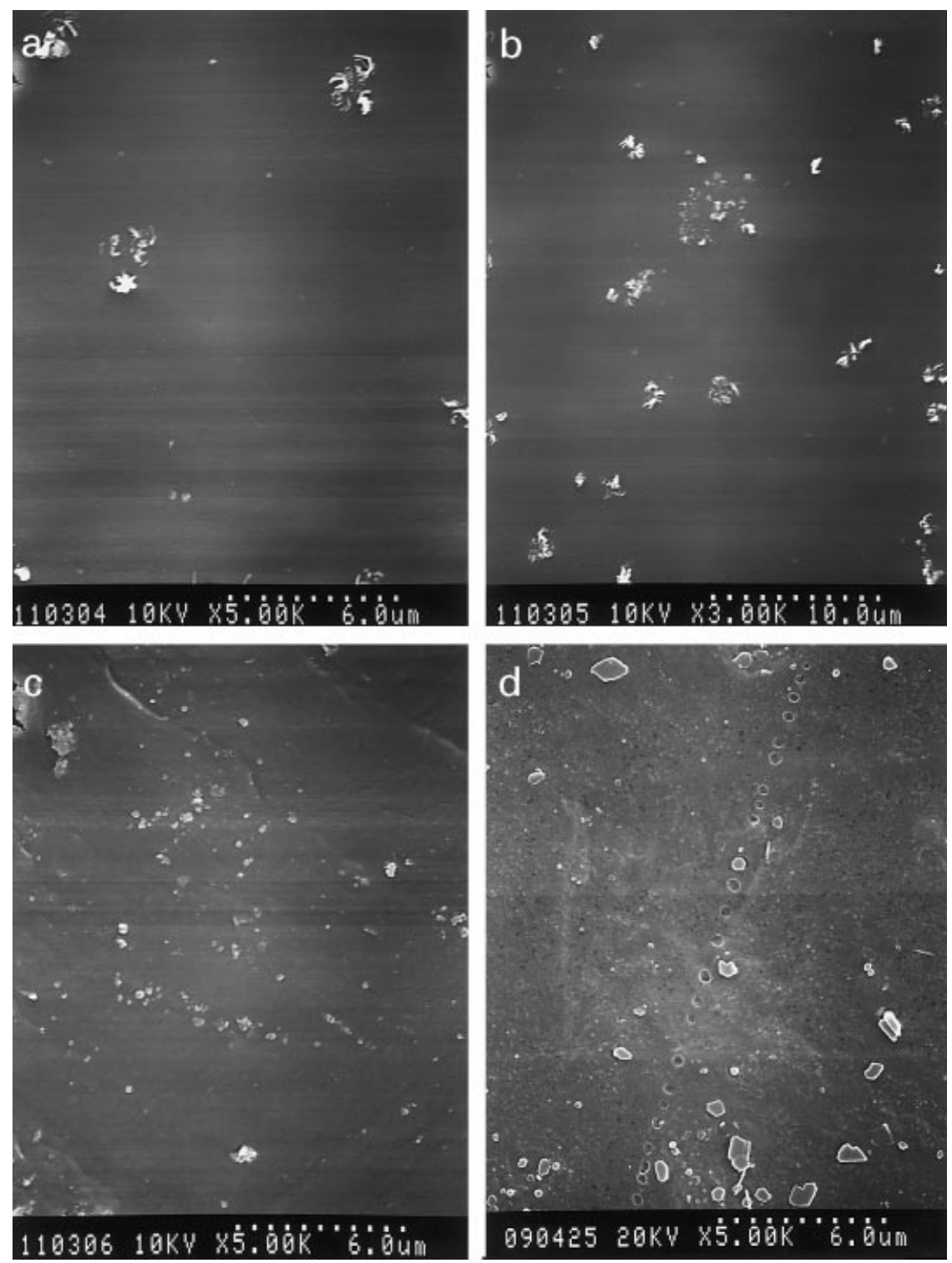

Fig. 5. SEM photographs of top surface of: (a) P-1; (b) P-2; (c) P-3; and (d) P-4 membranes.

In the formation of sublayer, precipitation of top layer created an additional barrier to mass transfer between the sublayer and the coagulation bath. If the top layer structure is relatively compact, it will increase the mass transfer resistance of solvent from the sublayer of casting solution to the coagulation bath. Accordingly, after the dense top layer of the M-1 membrane was formed, water outflow rate from the sublayer of casting solution to the coagulation bath declined. At this time, when the coagulant medium (ions) diffused through the top layer to work on its neighbouring solvent in the sublayer, the nucleus of polymer-poor phase occurred. Nucleus grew to form a pore when coagulant medium continually diffused into it to induce neighbouring solvent to diffuse into it. Thus, the polymer concentration of the polymer-rich phase surrounding the pore increased to form the pore wall. Although the action of the salt effect is highly localized, virtually all pores in the sublayer follow in this manner to some extent. Consequently, cellular morphologies occurred in the entire sublayer supporting the dense top layer during the precipitation process.

From the above two-step mechanism model, the ratio of coagulant medium inflow to solvent outflow is of utmost importance for the toplayer structure. A dense top layer is formed because solvent in the casting solution desolvates into the coagulation bath before the coagulant medium diffuses into the casting solution. The dense top layer stops growing when enough coagulant medium diffuses into the sublayer solution to create pores. On the other hand, if the coagulant medium diffuses into the casting solution before solvent in the casting solution desolvates into the coagulation bath, the coagulant medium may create pores in the membrane surface. In this work, only a small amount of dextran additive was effective to induce pore formation in the skin layer. The dextran additives are believed to change 

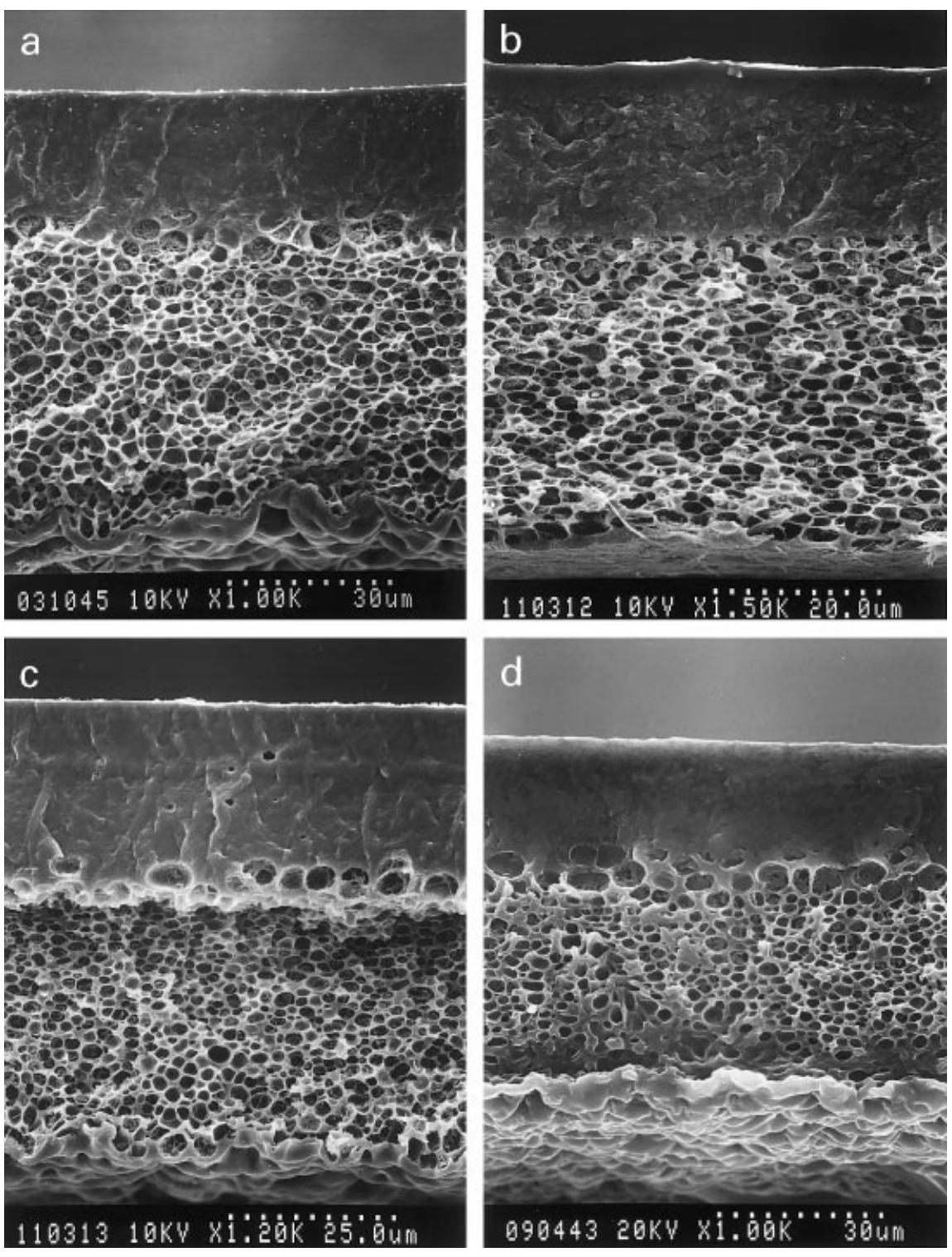

Fig. 6. SEM photographs of cross-section of: (a) P-1; (b) P-2; (c) P-3; and (d) P-4 membranes.

the diffusion rate of the coagulant medium. However, the PVA membrane formation mechanism is different from those traditional ternary systems consisting of solvent, nonsolvent and membrane forming polymer. The nonsolvent is a mixture of water, $\mathrm{Na}^{+}, \mathrm{K}^{+}, \mathrm{SO}_{4}^{-2}$ and $\mathrm{OH}^{-}$. Therefore, the addition of polymeric additives in the casting solution makes the analysis of the membrane formation mechanism more difficult. For simplicity, we return to a pseudo ternary system containing casting solution, coagulant medium and polymeric additive. Solvent is neglected since water is present in both the casting solution and the coagulation bath. Now, we discuss how the affinity between polymeric additive and casting solution as well as the affinity between polymeric additive and coagulant medium may explain the membrane structure based on the two-layer formation mechanism mentioned above. At first, we designed two experiments to measure the affinity between polymeric additive and casting solution as well as the affinity between polymeric additive and coagulant medium.

1. The affinity between polymeric additives and coagulant medium was investigated by the precipitation method. Polymeric additive $(0.28 \mathrm{~g})$ was dissolved in water $(1.72 \mathrm{~g})$ to form a solution. Then the solution was added to the coagulant medium $(10 \mathrm{~g})$. With different polymer additives, different results were observed. Dextran could be dissolved in the coagulant medium. Conversely, PVP aggregated to form a yellow liquid film floating on the coagulant medium. Apparently, dextran and coagulant medium indicate attractive interactions and compatibility, whereas PVP and coagulant medium indicate repulsive interactions and incompatibility. Therefore, the affinity between dextran and coagulant medium is higher than that between PVP and coagulant medium. 


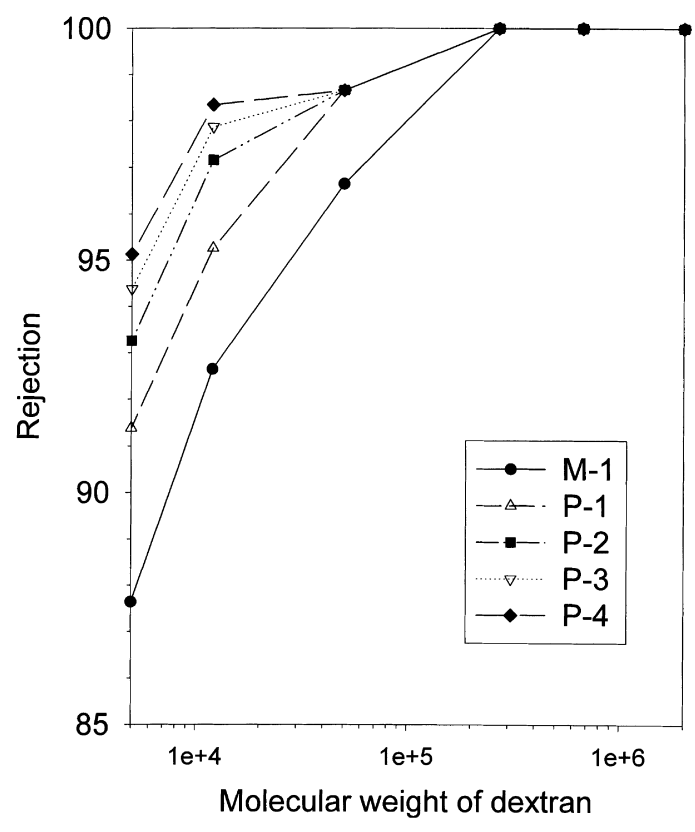

Fig. 7. Rejection curves of PVA membranes having various amounts of the PVP additive.

2. A specific amount of polymer additive was mixed with PVA $(1.4 \mathrm{~g})$ and water $(8.6 \mathrm{~g})$ to determine the affinity between polymeric additives and casting solution. The mixture was agitated at $90^{\circ} \mathrm{C}$ until a clear homogeneous solution was obtained. The solution was then placed in an isothermal thermostat, which was maintained at $25^{\circ} \mathrm{C}$, for a period of 3 days. The mixture maintained a homogeneous solution or phase-separated into a clear liquid phase

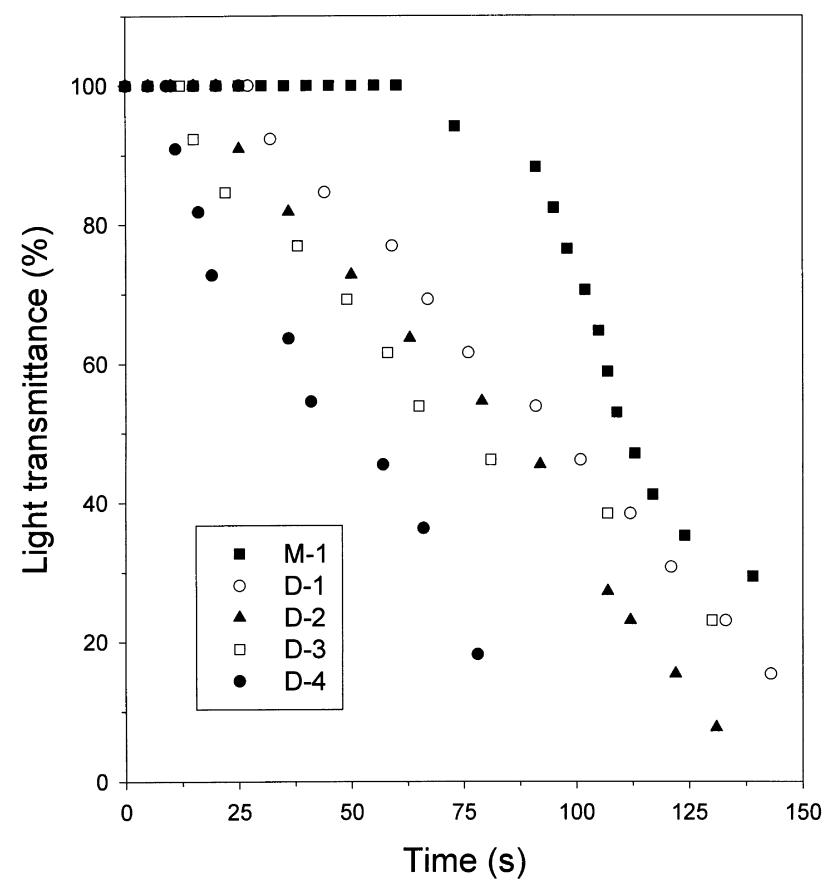

Fig. 8. The results of light transmission experiments of PVA membranes having various amounts of the dextran additive.

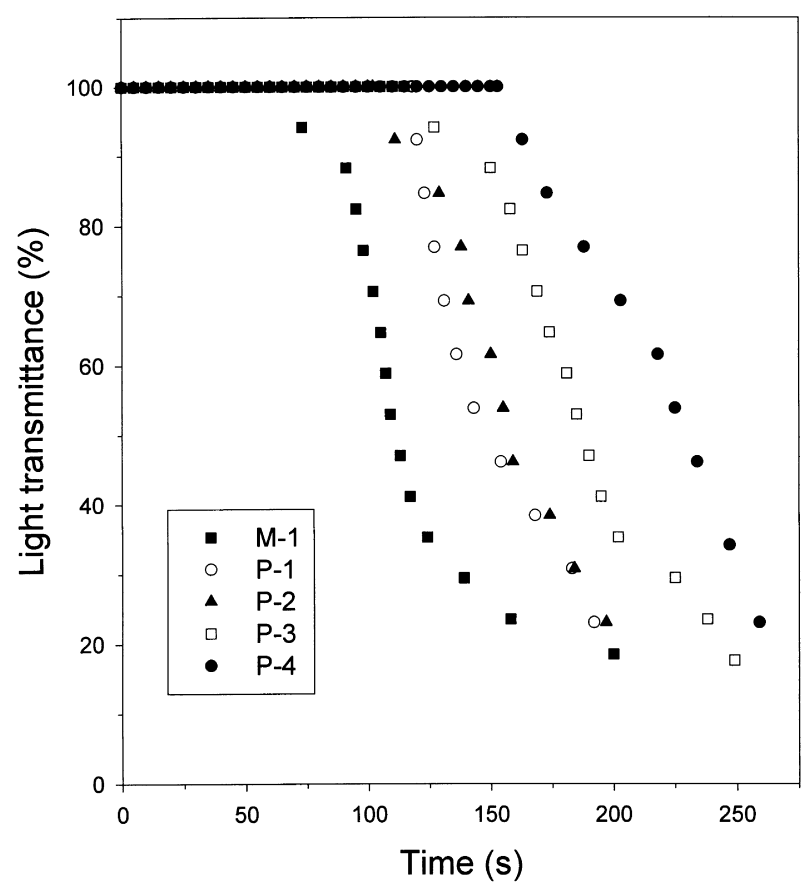

Fig. 9. The results of light transmission experiments of PVA membranes having various amounts of the PVP additive.

coexisting with a white solid. Phase separation easily occurred in the case of the addition of $0.5 \mathrm{~g}$ of dextran in the PVA solution. However, even though the addition of $1 \mathrm{~g}$ of PVP was not enough to bring about phase separation, indicating that a homogeneous solution could be observed. Therefore, it was concluded that PVP and dextran denotes high and low affinities with the casting solution, respectively. Of course, here the affinity between the two constituents has only relative meaning.

\subsection{Effect of dextran additive}

From Figs. 2 and 3, one can say that pores occur in the top layer and sublayer. This indicates that a casting solution with dextran additive is easier to phase separate to form pores during membrane formation. It is reasonable to attribute the fact that the coagulant medium could be attracted by the dextran in the casting solution. Therefore, the coagulant medium inflow increased to hasten the phase separation to induce pores in the top layer. This consists of the light transmission experiment, suggesting that the precipitation rate increased with increasing the amounts of dextran in the casting solution. In addition, after the coagulant medium diffuses into the casting solution, the coagulant medium could attract water to form the polymer-poor phase. In fact, phase separation in such a system involves the demixing of the polymer blends. Since the weak interaction between dextran and PVA in aqueous solution but the high affinity between dextran and coagulant medium, dextran might disperse heterogeneous at the casting solution and might even move to the polymer-poor phase. 


\subsection{Effect of $P V P$ additive}

Conversely, with the addition of PVP in the casting solution, the membrane structure was similar to that of the membrane without the additive present in the system. Especially, we could not distinguish the apparent difference of the structure of the top layer with or without the PVP additive present in the system from their SEM photographs. However, the filtration result from Fig. 7 indicates the compactness of the skin layer increased with increasing the amount of PVP in the casting solution. Since the dense top layer provides major resistance to the permeation of solute through the membrane, the microstructure of the top layer is strongly related to the effect of PVP additive though it is beyond the observable detection sensitivity of the SEM.

When the casting solution with PVP additive was immersed into the coagulation bath, the coagulant medium diffused into the casting solution with a slow rate due to the low affinity with PVP, in comparison with PVA membranes without PVP present in the system. This indicates that precipitation was delayed in the presence of PVP, which can be confirmed by the light transmission measurement. Furthermore, Cassu and Felisberti [16] have shown that PVA/PVP blends were miscible at every composition due to a hydrogen-bonding interaction between the PVA hydroxyl groups and the PVP carbonyl groups. In fact, the Flory-Huggins PVA-PVP interaction parameters were negative for all the compositions [17]. Therefore, strong interaction between PVP and PVA may contribute to the compact structure of the top surface in the membrane because it makes chain packing in the skin layer more probable. This fact can be associated with the larger mobility of PVP chains due to their lower molecular weight compared to the molecular weight of PVA, which allow better accommodation of PVP chains. Moreover, the precipitation rate decreased with increasing the amount of PVP in the casting solution so the PVA/PVP blend had a longer time to rearrange to effectively block the interstitial cavities within the top layer to generate a lower free volume and more compact structure before phase separation. This agrees with the fact that the membrane with the PVP additive restricts the permeation of dextran.

\section{Conclusion}

The results of the present work suggest that the addition of a small amount of appropriate polymeric additive can change the PVA membrane structure and permeability. A mechanism describing the affinity between additive and casting solution as well as between additive and coagulant medium was proposed to investigate the membrane structure in this complex system. The addition of dextran additive in the casting solution can enhance the inflow of coagulant medium because the affinity between dextran and coagulant medium is strong, while the addition of
PVP additive cannot. On the other hand, dextran favours to move the polymer-poor phase during phase separation due to the high affinity with coagulant medium and the low affinity with PVA in aqueous solution. Therefore, the addition of dextran additives can enhance the phase separation and induce pores in the top layer. Conversely, the PVP additives effectively block the interstitial cavities within the top layer to generate a more compact structure.

\section{Acknowledgements}

Acknowledgement is made to the donors of the Research Fund of Chinese Petroleum Company, administered by the National Science Council of the Republic of China.

\section{References}

[1] Loeb S, Sourirajan S. Sea water demineralization by means of an osmotic membrane. Adv Chem Ser 1963;38:117.

[2] Mulder M. Basic principles of membrane technology. Dordrecht: Kluwer Academic, 1991

[3] Sakurada I. Polyvinyl alcohol fibers. New York: Marcel Dekker, 1985.

[4] Kesting RE. Synthetic polymeric membranes. New York: Wiley, 1985.

[5] Young TH, Yao NK, Chang RF, Chen LW. Evaluation of asymmetric poly(vinyl alcohol) membranes for use in artificial islets. Biomaterials 1996;17:2139-45.

[6] Young TH, Chuang WY, Yao NK, Chen LW. Use of a diffusion model for assessing the performance of poly(vinyl alcohol) bioartificial pancreas. J Biomed Mater Res 1998;40:385-91.

[7] Cheng LP, Lin HY, Chen LW, Young TH. Solute rejection of dextran by EVAL membranes with asymmetric and particulate morphologies. Polymer 1998;39:2135-42.

[8] Young TH, Cheng LP, Lin HY. Interesting behavior for filtration of macromolecules through EVAL membranes. Polymer 2000;4:377-83.

[9] Lin FC, Wang DM, Lai CL, Lai JY. Effect of surfactants on the structure of PMMA membranes. J Membrane Sci 1997;123:281-91.

[10] Reuvers AJ, van der Berg JWA, Smolders CA. Formation of membranes by means of immersion precipitation. Part I. A model to describe mass transfer during immersion precipitation. J Membrane Sci 1987;34:45-65.

[11] Smolders CA, Reuvers AJ, Boom RM, Wienk IM. Microstructures in phase-inversion membranes. 1. Formation of macrovoids. J Membrane Sci 1992;73:259-75.

[12] Kobayashi T, Miyamoto T, Nagai T, Fujii N. Polyacrylonitrile ultrafiltration membranes containing negatively charged groups for permeation and separation of dextran and dextransulfate. J Appl Polym Sci 1994;52:1519-28.

[13] Strathmann H, Kock K, Amar P. The formation mechanism of asymmetric membranes. Desalination 1975;16:179-203.

[14] Kang YS, Kim HJ, Kim UY. Asymmetric membrane formation via immersion precipitation method. I. Kinetic effect. J Membrane Sci 1991;60:219-32.

[15] Young TH, Chen LW. A two step mechanism of diffusion-controlled ethylene vinyl alcohol membrane formation. J Membrane Sci 1991;57:69-81.

[16] Cassu SN, Felisberti MI. Poly(vinyl alcohol) and poly(vinyl pyrrolidone) blends: miscibility, microheterogeneity and free volume change. Polymer 1997;38:3907-11.

[17] Cassu SN. MS thesis, University Estadual d Campinas, CampinasBrasil, 1995. 